\title{
Subolesin vaccination inhibits blood feeding and reproduction of Haemaphysalis longicornis in rabbits
}

Seung-Hun Lee ${ }^{1,2 \dagger}$, Jixu Li ${ }^{1 \dagger}$, Paul Franck Adjou Moumouni ${ }^{1}$, Kiyoshi Okado ${ }^{1}$, Weiqing Zheng ${ }^{1}$, Mingming Liu', Shengwei $\mathrm{Ji}^{1}$, Soochong Kim², Rika Umemiya-Shirafuji ${ }^{* *}$ and Xuenan Xuan ${ }^{1 *}$

\begin{abstract}
Background: Ticks can transmit numerous tick-borne pathogens and cause a huge economic loss to the livestock industry. Tick vaccines can contribute to the prevention of tick-borne diseases by inhibiting tick infestation or reproduction. Subolesin is an antigenic molecule proven to be a potential tick vaccine against different tick species and even some tick-borne pathogens. However, its effectivity has not been verified in Haemaphysalis longicornis, which is a widely distributed tick species, especially in East Asian countries. Therefore, the purpose of this study was to evaluate the effectivity of subolesin vaccination against $H$. longicornis in a rabbit model.
\end{abstract}

Methods: Haemaphysalis longicornis (Okayama strain, female, adult, parthenogenetic strain) and Japanese white rabbits were used as the model tick and animal, respectively. The whole open reading frame of H. longicornis subolesin (HISu) was identified and expressed as a recombinant protein using E. coli. The expression was verified using sodium dodecyl sulfate polyacrylamide gel electrophoresis, and the immunogenicity of rHISu against anti-H. longicornis rabbit serum was confirmed using Western blotting. After vaccination of rHISu in rabbits, experimental infestation of H. longicornis was performed. Variables related to blood-feeding periods, pre-oviposition periods, body weight at engorgement, egg mass, egg mass to body weight ratio, and egg-hatching periods were measured to evaluate the effectiveness of subolesin vaccination.

Results: The whole open reading frame of HISu was $540 \mathrm{bp}$, and it was expressed as a recombinant protein. Vaccination with rHISu stimulated an immune response in rabbits. In the rHISu-vaccinated group, body weight at engorgement, egg mass, and egg mass to body weight ratio were statistically significantly lower than those in the control group. Besides, egg-hatching periods were extended significantly. Blood-feeding periods and pre-oviposition periods were not different between the two groups. In total, the calculated vaccine efficacy was $37.4 \%$.

Conclusions: Vaccination of rabbits with rHISu significantly affected the blood-feeding and reproduction in $\mathrm{H}$. longicornis. Combined with findings from previous studies, our findings suggest subolesin has the potential to be used as a universal tick vaccine.

Keywords: Haemaphysalis longicornis, Subolesin, Vaccine, Tick, Akirin, Tick-borne disease

*Correspondence: umemiya@obihiro.ac.jp; gen@obihiro.ac.jp

† Seung-Hun Lee and Jixu Li contributed equally to this work

${ }^{1}$ National Research Center for Protozoan Diseases, Obihiro University

of Agriculture and Veterinary Medicine, Obihiro, Hokkaido 080-8555, Japan

Full list of author information is available at the end of the article

\section{Background}

Ticks are one of the most important vectors and can transmit numerous pathogens covering bacteria, viruses, and parasites including Anaplasma phagocytophilum, Borrelia burgdorferi, Babesia spp., Theileria spp., severe

c) The Author(s) 2020. This article is licensed under a Creative Commons Attribution 4.0 International License, which permits use, sharing, adaptation, distribution and reproduction in any medium or format, as long as you give appropriate credit to the original author(s) and the source, provide a link to the Creative Commons licence, and indicate if changes were made. The images or other third party material in this article are included in the article's Creative Commons licence, unless indicated otherwise in a credit line to the material. If material is not included in the article's Creative Commons licence and your intended use is not permitted by statutory regulation or exceeds the permitted use, you will need to obtain permission directly from the copyright holder. To view a copy of this licence, visit http://creativecommons.org/licenses/by/4.0/. The Creative Commons Public Domain Dedication waiver (http://creativecommons.org/publicdomain/zero/1.0/) applies to the data made available in this article, unless otherwise stated in a credit line to the data. 
fever with thrombocytopenia syndrome (SFTS) virus, and tick-borne encephalitis virus [1]. By transmitting tick-borne diseases, ticks cause huge economic loss to the livestock industry. A heavy tick infestation in animals, even without disease transmission, can cause severe blood loss, resulting in economic problems for breeding farms [2]. Therefore, the control of ticks and tick-borne disease is important in the fields of agriculture and veterinary medicine.

Haemaphysalis longicornis is a widely distributed tick species. It is the dominant tick species in Korea and plays an important role in East Asian countries, including China and Japan [3, 4]. Recently, H. longicornis invaded North America, raising public attention [5]. Based on recent studies, $H$. longicornis is a proven vector of tickborne pathogens including SFTS virus and Babesia gibsoni $[6,7]$. Unfortunately, there are no effective vaccines against both tick-borne pathogens. Tick-borne pathogens can be transmitted transstadially and transovarially [6, 8], which means an infected tick can reproduce to create plentiful infected offspring. Therefore, the prevention of both transstadial and transovarial transmission is important to prevent the spread of tick-borne diseases.

Thus far, there have been efforts to prevent tick-borne diseases in different ways, e.g. using tick-resistant breeds, treatment with acaricides, and development of vaccines [9]. However, widespread treatment with acaricides is limited by factors such as safety concerns, environmental pollution, and the existence of acaricide-resistant tick strains that puts the effectiveness of acaricides in doubt [9]. Regarding vector-borne diseases such as yellow fever, malaria, and theileriosis, many studies have tried to develop vaccines against them; however, a lot of efforts still are required to reach the goal [10-12]. In addition, aiming to control each pathogen entails a huge cost, requires a large workforce and long periods. To overcome these disadvantages, researchers have aimed to develop vaccines against vectors and not for individual pathogens. One of the most well-known tick vaccines is the Bm86-based formulation derived from Rhipicephalus (Boophilus) microplus gut protein [13]. The Bm86-based vaccination is cost-effective and more environmentally friendly than acaricides; however, the Bm86-based vaccines are ineffective against some tick species $[14,15]$. In addition, homologs of Bm86 were not effective against other tick species such as Ixodes ricinus and Amblyomma cajennense [16, 17]. Moreover, the effectiveness of Bm86based vaccination has not been proven in Haemaphysalis spp. [18], which makes it difficult to be used as a universal tick vaccine.

Subolesin is an antigenic molecule and was first identified in Ixodes scapularis in 2003 [19]. Subsequent studies revealed that the function of subolesin is related to tick blood-feeding and reproduction using RNAi treatment [20]. Subolesin was initially identified in hard ticks and was subsequently revealed as an ortholog of akirin in insects and vertebrates [21, 22]. Recent studies have shown that this molecule has the potential to be used as a vaccine against different tick species in different hosts. Additionally, the vaccination gives protection against tick-borne pathogens, including Borrelia burgdorferi, Anaplasma marginale and Babesia bigemina [23-25].

Thus far, subolesin has been studied mainly in species of Ixodes, Rhipicephalus and Dermacentor [24, 26]. Regarding Haemaphysalis spp., Rahman et al. [27] used RNAi treatment to demonstrate the existence of subolesin in H. longicornis and showed that it has an identical function in different tick species. However, the sequence information was defective and not verified in animal experiments. As RNAi treatment and animal experiments do not always show identical results [28, 29], it is essential to evaluate the effectivity of vaccination in animal experiments. Therefore, the purpose of this study was to evaluate the effectivity of subolesin vaccination in $H$. longicornis as a tick vaccine in rabbits.

\section{Methods}

\section{Ticks and rabbits}

In this study, parthenogenetic H. longicornis (Okayama strain) was used as the model tick. The tick has been maintained as a colony by routine passage in Japanese white rabbits at the National Research Center for Protozoan Diseases, Obihiro University of Agriculture and Veterinary Medicine, Japan [30]. Four-to-eight-monthold female ticks were used in this study. Before the start of blood-feeding, the ticks were habituated at room temperature with $40 \%$ humidity for two weeks. For experimental infestation, female ticks derived from the same batch were used, to reduce the potential bias caused by batch differences.

Japanese white rabbits (specific-pathogen-free animal, female, 9-weeks-old) were purchased from a company (Clea Japan, Tokyo, Japan). The rabbits were housed until 3-8-months-old in a room with a temperature of $25^{\circ} \mathrm{C}$, humidity of $40 \%$, and controlled lighting (i.e. period of light from 6:00 to 19:00 h). The rabbits had access to tap water and commercial pellets (CR-3; CLEA Japan, Tokyo) ad libitum throughout the experiments.

\section{Identification of subolesin in $\mathrm{H}$. longicornis}

Total RNA was extracted from $H$. longicornis using the Direct-zol RNA Miniprep (Zymo Research, Irvine, CA, USA) after breaking the tick mechanically with liquid nitrogen. cDNA was synthesized using the PrimeScript ${ }^{\mathrm{TM}}$ 
RT-PCR Kit (Takara, Shiga, Japan), according to the manufacturer's protocols. The extracted cDNA was kept at $-30{ }^{\circ} \mathrm{C}$ until further experiments were performed.

Primers were newly designed to amplify the whole open reading frame (ORF) of $H$. longicornis subolesin $(\mathrm{HISu})$. The primers were designed based on the previously reported sequences of subolesin in other tick species in the GenBank database and cDNA library of $H$. longicornis in our laboratory [31]. Using the primers, polymerase chain reaction (PCR) was performed as per the following cycles: initial denaturation at $94^{\circ} \mathrm{C}$ for 5 min, followed by 35 cycles of $94{ }^{\circ} \mathrm{C}$ for $30 \mathrm{~s}, 50{ }^{\circ} \mathrm{C}$ for $30 \mathrm{~s}, 72{ }^{\circ} \mathrm{C}$ for $30 \mathrm{~s}$, and a final extension step at $72{ }^{\circ} \mathrm{C}$ for $5 \mathrm{~min}$. The primers used in this study are listed in Table 1.

\section{Cloning, sequencing, and phylogenetic analysis of HISu}

The amplified PCR product was ligated to pGEM-T Easy Vector (Promega, Tokyo, Japan) and was transformed into Escherichia coli DH5 $\alpha$. It was cultured on a LuriaBertani agar plate containing $100 \mu \mathrm{g} / \mathrm{ml}$ ampicillin at $37{ }^{\circ} \mathrm{C}$. Finally, five colonies were selected and cultured for plasmid extraction and sequencing. The extracted plasmids were sequenced using the BigDye Terminator v3.1 Cycle Sequencing Kit (Applied Biosystems, New York, USA), and the results were analyzed using the ABI PRISM 3100 Genetic Analyzer (Applied Biosystems, Carlsbad, CA) according to the manufacturer's instructions. All the plasmids were sequenced bidirectionally and were aligned.

The obtained sequence was analyzed by BLAST to compare its identity with that of other subolesin sequences. Phylogenetic analysis was performed to analyze the molecular relationship among subolesin identified in insects. The phylogenetic tree was constructed using MEGA 7.0. using the Maximum-Likelihood method and 500 replications [5].

The sequence and molecular weight of $\mathrm{rHISu}$ were deduced using a plasmid Editor v2.0.61.

\section{Recombinant HISu (rHISu) expression and purification}

The whole ORF of HISu was inserted into a pCold ProsS2 vector (TaKaRa, Japan), which has a His- and ProS2-tag, for expression as a recombinant protein. To insert HISu into the vector, restriction enzyme sites (XhoI for the upstream site and EcoRI for the downstream site) were added using PCR with the primers listed in Table 1. The inserted expression vector was transformed into $E$. coli BL21(DE3), and recombinant protein was expressed according to the vector manufacturer's protocols. In brief, the transformed E. coli BL21(DE3) was cultured at $37{ }^{\circ} \mathrm{C}$ with vigorous shaking until the optical density at $600 \mathrm{~nm}$ reached 0.4 , and was cooled to $15^{\circ} \mathrm{C}$ for $30 \mathrm{~min}$.
After that, induction was initiated with isopropyl $\beta$-D-1thiogalactopyranoside concentration of $1.0 \mathrm{mM}$ and was cultured at $15{ }^{\circ} \mathrm{C}$ along with shaking for $24 \mathrm{~h}$.

The cultured $E$. coli was washed with phosphate-buffered saline (PBS) three times and broken up using sonification. The recombinant HISu was purified using His GraviTrap (GE Healthcare, Tokyo, Japan) according to the manufacturer's protocols. In brief, the column was equilibrated with $10 \mathrm{ml}$ binding buffer $(20 \mathrm{mM}$ sodium phosphate, $500 \mathrm{mM}$ sodium chloride, $20 \mathrm{mM}$ imidazole, $\mathrm{pH}$ 7.4), and samples were applied. After washing the column with the binding buffer two times, the protein was eluted with $3 \mathrm{ml}$ of the elution buffer $(20 \mathrm{mM}$ sodium phosphate, $500 \mathrm{mM}$ sodium chloride, $500 \mathrm{mM}$ imidazole, pH 7.4).

Only the soluble fraction was used for further experiments. Expression and purification of rHISu were verified using sodium dodecyl sulfate polyacrylamide gel electrophoresis (SDS-PAGE). SDS-PAGE results were visualized by Coomassie blue staining. The protein concentration was estimated by using Pierce BCA Protein Assay Kit (Thermo Fisher Scientific, Tokyo, Japan).

\section{Production of anti-H. longicornis rabbit serum}

To produce anti- $H$. longicornis rabbit serum, a rabbit was experimentally infested with $H$. longicornis using the ear bag method, as previously described [32]. Experimental infestation was performed three times; each time, the rabbit was infested with 40 female ticks (20 ticks for each ear), with two-week intervals between infestations. Two weeks after the last infestation, serum was collected from the rabbit.

\section{Western blotting}

The immune response of $\mathrm{rHISu}$ against $H$. longicornis infestation was confirmed using Western blotting. In brief, $0.03 \mu \mathrm{g} \mathrm{rHISu}$ was separated using SDS-PAGE and transferred to an Amersham Protran 0.45 nitrocellulose Western blotting membrane (GE Healthcare, Tokyo, Japan). After blocking with TBS containing $0.1 \%$ Tween 20 (TBS-T) and 3\% skimmed milk, the membranes were probed with anti- $H$. longicornis rabbit serum (described above) or naïve rabbit serum diluted with TBS-T at a ratio of 1:600. HRP-conjugated goat anti-rabbit IgG $(\mathrm{H}+\mathrm{L})$ cross-adsorbed secondary antibody (Thermo Fisher Scientific) was used as a secondary antibody diluted with TBS-T at a ratio of 1:5000. Finally, the reaction was visualized using Amersham ECL Prime Western Blotting Detection Reagent (GE Healthcare, Tokyo, Japan). The results were scanned using a VersaDoc ${ }^{\mathrm{TM}}$ imaging system (Bio-Rad Laboratories, Tokyo, Japan). 
To estimate the immunogenicity of rHlSu after vaccination, Western blotting was similarly performed using rHISu and rHISu-immunized rabbit serum (described below), except that serum dilution was done at 1:2000.

\section{Vaccine trial}

To evaluate the efficacy of rHISu as a tick vaccine, two groups were established (vaccination group and PBS group). Each group included three Japanese white rabbits. Before vaccination, the purified $\mathrm{rHISu}$ protein was dialyzed with PBS using Pierce Protein Concentrator 30K MWCO (Thermo Fisher Scientific) according to the manufacturer's recommendation. In brief, $\mathrm{rHISu}$ in the elution buffer was mixed with $3 \mathrm{ml}$ of PBS and was centrifuged at $8000 \times g$ leaving a residual $1 \mathrm{ml}$ buffer. Again, the remaining buffer was centrifuged in the same way with $5 \mathrm{ml}$ of PBS.

rHISu or PBS was then conjugated with adjuvant (TiterMax Gold, Sigma-Aldrich, Japan). In total, $500 \mu \mathrm{g}$ of $\mathrm{rHISu}$ (or the same amount of PBS for the control group) was mixed with $500 \mu \mathrm{l}$ of the adjuvant and was intradermally injected into the back of each rabbit. The dose was determined according to the manufacturer's instructions and based on the body weight of rabbits. The vaccination was boosted twice at two-week intervals. Before each vaccination, blood was collected from the ear vein to obtain sera, and antibody levels were evaluated using enzyme-linked immunosorbent assay (ELISA).

\section{ELISA protocol}

Antibody titers in the sera were evaluated using ELISA. In brief, purified rHISu $(0.25 \mu \mathrm{g}$ per well) in $50 \mathrm{mM}$ carbonate-bicarbonate buffer was used to coat ELISA plates and left overnight at $4{ }^{\circ} \mathrm{C}$. Blocking of the plate was performed with $100 \mu \mathrm{l}$ of $3 \%$ skimmed milk-PBS at $37^{\circ} \mathrm{C}$ for one hour. Next, $50 \mu \mathrm{l}$ sera diluted at a ratio of 1:100 in 3\% skimmed milk-PBS were incubated at $37^{\circ} \mathrm{C}$ for one hour. After washing the plate with PBS containing 0.1\% Tween 20 (PBS-T) six times, $50 \mu \mathrm{l}$ of HRP-conjugated goat antirabbit IgG diluted at a ratio of 1:4000 with $3 \%$ skimmed milk-PBS, was added to each well and incubated at 37 ${ }^{\circ} \mathrm{C}$ for one hour. After washing the plate with PBS-T six times, $100 \mu$ l of substrate solution (ABTS, Roche, Tokyo,
Japan) was added to each well and kept in the dark for one hour. Optical density value was measured three times at $415 \mathrm{~nm}$ using a spectrometer (Corona microplate reader MTP-500, Corona Electric, Ibaraki, Japan).

\section{Effect of vaccination}

Three weeks after the last vaccination, experimental infestation with female ticks was performed as described above. After starting the experimental infestation, the condition of ticks and rabbits was checked daily. The ticks that detached abnormally after the start of feeding (detachment without external force, tick's body weight $<50 \mathrm{mg}$ ), were excluded from the data analysis. During the experimental infestation, five and six ticks in the vaccination group and PBS group, respectively, were excluded from the data analysis due to abnormal detachment. After blood-feeding, the fully engorged ticks were incubated in the dark at $25^{\circ} \mathrm{C}$ and saturated humidity for oviposition.

The effect of vaccination was evaluated based on the following factors: blood-feeding period (days), pre-oviposition period (days), body weight at engorgement (mg), egg mass at 10 days after oviposition (mg), egg mass to body weight ratio, and egg period (days). Each parameter was estimated daily. In total, vaccine efficacy was calculated by the formula: $(1-$ reduction ratio of body weight $\times$ reduction ratio of egg mass) $\times 100$.

\section{Statistical analysis}

Differences between groups were analyzed using Student's t-test or the Mann-Whitney test according to the distribution of data using SPSS Statistics for Windows, Version 26.0 (IBM Crop., Armonk, NY, USA). A P-value of less than 0.05 was considered statistically significant.

\section{Results \\ Identification and phylogenetic analysis of HISu}

On cloning and sequencing, the whole ORF of HISu was $540 \mathrm{bp}$ and the sequence length was identical with that of subolesin in $H$. flava and $H$. punctata, but differed from that in H. elliptica (528 bp). Based on a BLASTN search, $\mathrm{HISu}$ showed the highest genetic similarity to subolesin in Haemaphysalis elliptica at 93.7\% (JX193850). Among

Table 1 Primers used in this study

\begin{tabular}{|c|c|c|}
\hline Name & Sequence $\left(5^{\prime}-3^{\prime}\right)^{a}$ & Purpose \\
\hline HISu_For & ATGGCTTGTGCGACATTAAAG & \multirow{2}{*}{$\begin{array}{l}\text { Identification and amplification of } \\
\text { H. longicornis subolesin }\end{array}$} \\
\hline HISu_Rev & TTATGACAAATAGCTTGGAGTGGC & \\
\hline HISu_Xhol_For & ctcgagATGGCTTGTGCGACATTAAA & Insertion of restriction enzyme site \\
\hline HISu_EcoRI_Rev & gaattcTTATGACAAATAGCTTGGAGTGGC & \\
\hline
\end{tabular}

a Restriction enzyme sites are in lower case 
Haemaphysalis spp., the similarity ranged from $90.0 \%(H$. punctata, DQ159972) to 93.7\% (H. elliptica, JX193850). At the amino acid level, it shows the highest similarity with $H$. elliptica at $96.1 \%$ (AGI44626, JX193850) by a BLASTP search.

Based on the phylogenetic analysis, subolesin is grouped in a monophyletic clade with other Haemaphysalis orthologs including Dermacentor, Hyalomma, Rhipicephalus and Amblyomma. Subolesin of H. longicornis showed the closest relationship with the subolesin in $H$. elliptica, followed with that of $H$. flava and $H$. punctata (Fig. 1). The obtained sequence was submitted to the GenBank database (accession number: MT199422).

\section{rHISu expression, purification, and immunogenicity}

The $\mathrm{rHISu}$ was expressed at approximately $44 \mathrm{kDa}$ (tag size, $\sim 23 \mathrm{kDa}$ ) as expected (Fig. 2a). After purification, the dominant band at the target size remained; thereafter, only purified $\mathrm{rHlSu}$ was used in further experiments. Using Western blotting, the immune response of rHISu was verified (Fig. 2b). At the target size, reactivity was observed in the $H$. longicornis-infested rabbit serum and rHISu-immunized rabbit serum, but not in naïve serum.

\section{Vaccination and immune response}

After the first vaccination, antibody levels in the vaccination group were significantly elevated to three to four times more than that in the PBS group (Mann-Whitney U-test: $U=0.000, Z=-3.576, P<0.0001)$. The antibody levels did not increase further after the second and third vaccination (Fig. 3). Antibody levels in the PBS group did not differ statistically and were elevated when compared to naïve rabbit serum before and after the second immunization, respectively.

\section{Effect of vaccination against $H$. longicornis}

In the rHISu-vaccinated group, body weight at engorgement (Mann-Whitney U-test: $U=4117.000$, $Z=-4.780, P<0.0001)$, egg mass at 10 days after oviposition (t-test: $t=-7.139, P<0.0001$ ), and egg mass to body weight ratio (t-test: $t=-5.840, P<0.0001$ ) were significantly lower than those in the PBS group (Fig. 4; Additional file 1: Table S1). The egg-hatching periods were significantly longer in the vaccinated group than in the PBS group (Mann-Whitney U-test: $U=4627.500$, $Z=-2.432, P=0.015)$. In cases of body weight and egg mass, the vaccinated group had values that were $12.0 \%$ and $28.9 \%$ lower than those in the PBS group, respectively. The calculated vaccine efficacy was $37.4 \%$. Bloodfeeding periods (Mann-Whitney U-test: $U=5922.000$, $Z=-1.223, P=0.221$ ) and pre-oviposition periods
(Mann-Whitney U-test: $U=6076.000, Z=-0.417$, $P=0.676$ ) were not different between the two groups.

\section{Discussion}

Subolesin and its orthologue akirin were identified in different insects, and their molecular information has been analyzed [21, 22]. However, only partial sequence information is available on $H$. longicornis (EU289292), which remains unpublished. As the essential and effective epitope of subolesin has not yet been confirmed [33], this study was initiated to identify and express the whole ORF of $\mathrm{HISu}$ in order to use the entire region as a recombinant protein.

Previous studies have shown that subolesin-based vaccination showed effectivity as a tick vaccine in cattle, rabbits, and mice based on different parameters including body weight, mortality, molting, tick infestation, fertility, and oviposition [22, 25, 26, 34]. Moreover, vaccination also reduced the level of infection with tick-borne pathogens [23-25]. The vaccine efficacy in this study was $37.4 \%$, which was calculated under consideration of the body weight and egg mass. As previous studies were performed under various experimental conditions, such as during the developmental stage; with different tick species, hosts, or adjuvants; and methods to calculate efficacy, it is difficult to directly compare the efficacy of subolesin-based vaccination between studies. In summary, previous studies showed that subolesin-based vaccination protects against tick infestation.

In this study, subolesin vaccination significantly reduced body weight, egg mass, and the ratio of egg mass to body weight $(P<0.0001)$. This result is consistent with the results of previous studies and the original function of subolesin, which indicates that vaccination has the same effectivity as RNAi treatment. In addition, the significant decrease in the egg mass to body weight ratio indicates that both are affected by vaccination, and the effect was more significant on egg mass. Interestingly, egg-hatching periods were extended significantly in the vaccinated group $(P=0.015)$. To the best of our knowledge, this study is the first to show that subolesin affects not only blood-feeding and egg mass but also the egg-hatching period. However, additional assessments are required to confirm that subolesin vaccination has the same function in other tick species.

This study evaluated blood-feeding periods because subolesin affects blood-feeding and may be affected by short blood-feeding periods. However, there was no difference between the two groups. Moreover, the pre-oviposition period was also not different.

Tick vaccines can control ticks by affecting different factors such as mortality, molting, number of infested ticks, fertility, and oviposition. Contreras et al. [26] 
showed that a higher mortality was observed in a subolesin-vaccinated group than in a control group; however, a difference in mortality was not observed in this study. In this study, some ticks abnormally detached after they started feeding; however, the number of detached ticks did not differ between the two groups. Since the model ticks were adults in this study, we focused on factors related to blood-feeding and reproduction. Additional studies are required to assess the effect of subolesinbased vaccination on the mortality of eggs and molting of $H$. longicornis in the larval and nymph stages.

In this study, the antibody levels reached their highest after the first vaccination and appeared to be sustained for more than two months (Fig. 3). Even after experimental infestation, the antibody levels did not increase further. Similar trends were identified after diluting the serum up to 1:80,000 (Additional file 1: Figure S1). According to previous studies, the effectiveness of vaccination is related to antibody levels against subolesin in the host $[22,35]$. Based on the current vaccine formula, a one-time vaccination might be enough to obtain the objective antibody levels in the host. As $H$. longicornis is a three-host tick and has a longer life-cycle than $R$. microplus, which is a one-host tick, this vaccination can effectively control $H$. longicornis in endemic areas during the prevailing season, with a fewer number of vaccinations.

In this study, rHlSu was used for vaccination without removing the tags (His and ProS2 tag). Owing to the remaining tags, we cannot exclude the possibility of a non-specific immune response. However, based on the previous studies, subolesin accompanying different tags such as His-, Trx- and S-tag showed consistent vaccination results $[22,34]$. Therefore, we suppose that even if there is a non-specific immune response, the response has minor effects on the experiment.

\section{Conclusions}

Vaccination of rabbits with rHISu significantly reduced body weight, egg mass, and egg mass to body weight ratio in $H$. longicornis. In addition, egg-hatching periods were also significantly extended. Finally, the vaccine efficacy was $37.4 \%$. This finding raises the possibility that vaccination may gradually decrease the number of offspring. Variables related to the blood-feeding and preoviposition periods were not different between groups. In conclusion, this study showed the efficacy of $\mathrm{rHISu}$ as a tick vaccine against $H$. longicornis in rabbits. To our

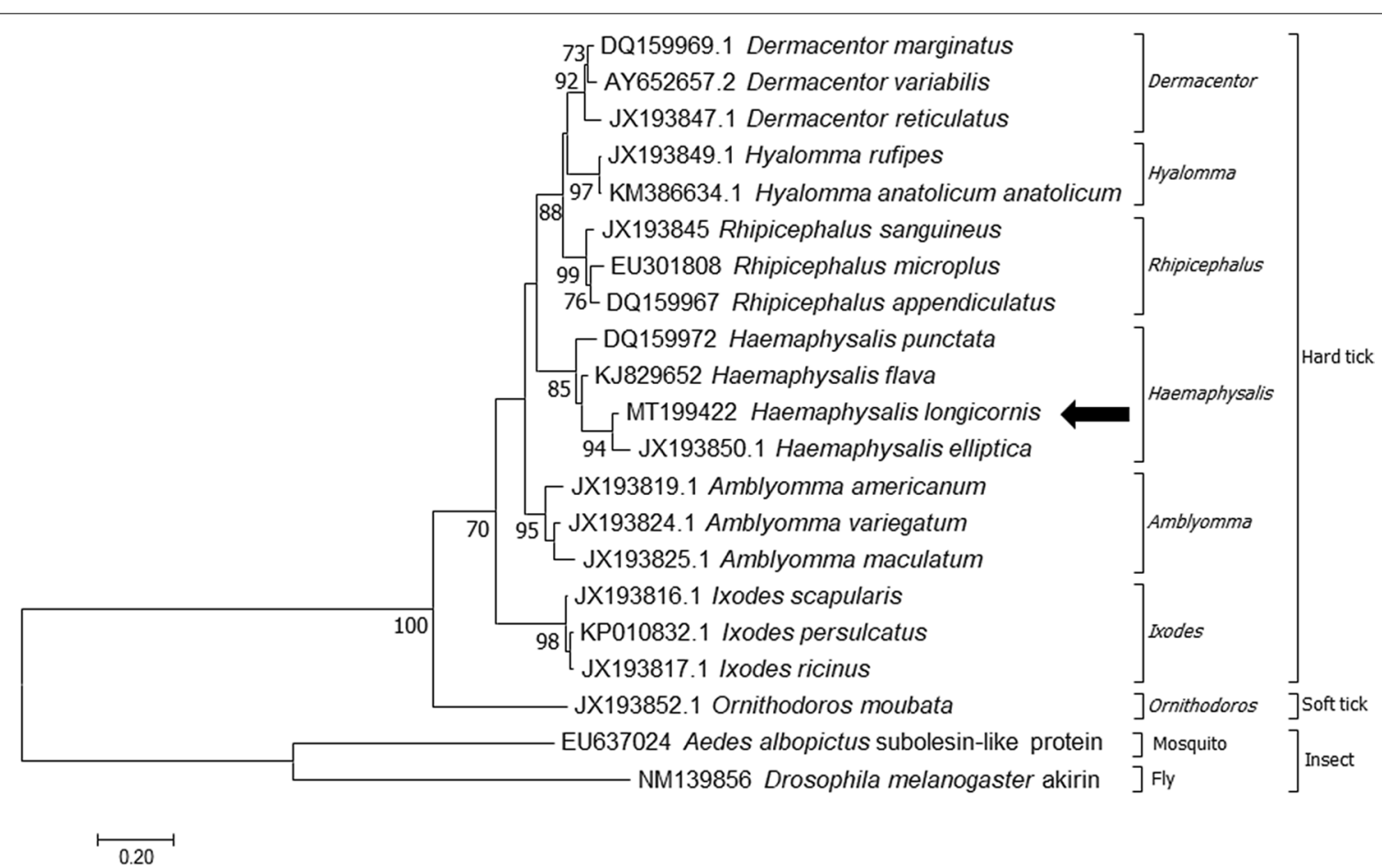

Fig. 1 The phylogenetic relationships of subolesin identified in this study. For this analysis, other subolesin sequences were obtained from the GenBank database. The tree is generated based on the Maximum-Likelihood method with 500 replications using MEGA 7.0. The sequence obtained in this study is indicated by an arrow. Bootstrap values $<70$ were omitted 


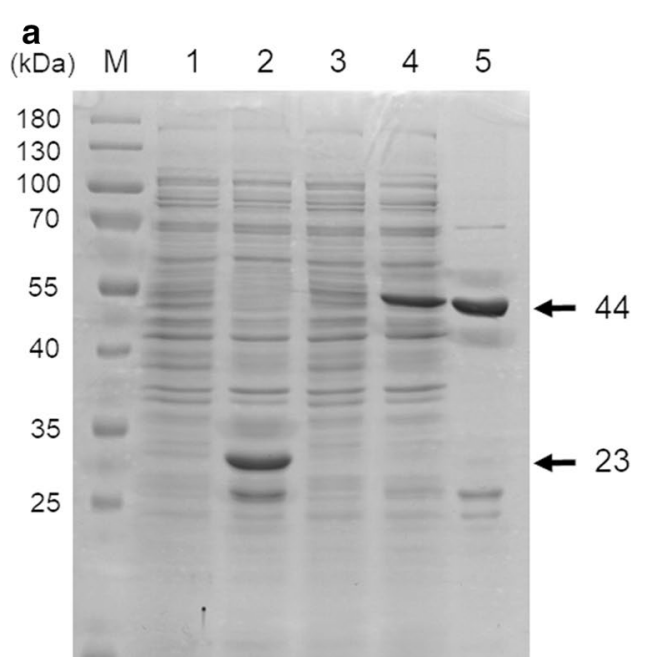

\section{b}

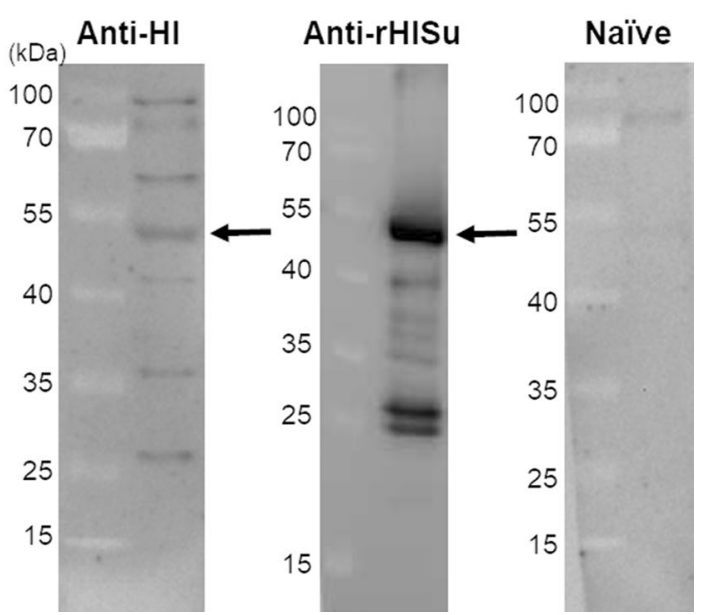

Fig. 2 Expression and immune response of rHISU. a Expression and purification of recombinant Haemaphysalis longicornis subolesin (rHISu) using an E. coli expression system. Each protein (Lanes 1-4,6 $\mu \mathrm{g}$; Lane 5, $2.25 \mu \mathrm{g}$ ) was applied and separated by SDS-PAGE. Lane M: protein marker; Lane 1, empty vector without IPTG induction; Lane 2, empty vector with IPTG induction; Lane 3, vector including subolesin without IPTG induction; Lane 4, vector including subolesin with IPTG induction; Lane 5, purified rHISu. b The immune response of rHISu was verified using Western blotting. Purified rHISu $(0.03 \mu \mathrm{g})$ was used for Western blotting. Serum dilutions were 1:600 for lanes anti-H. longicornis serum and Naïve serum, and 1:2000 for lane anti-rHISu serum. Secondary antibody dilution was 1:5000 for all lanes. Anti-HI, reaction with anti-H. longicornis serum; Anti-rHISu, reaction with anti-rHISu serum; Naïve, reaction with naïve serum

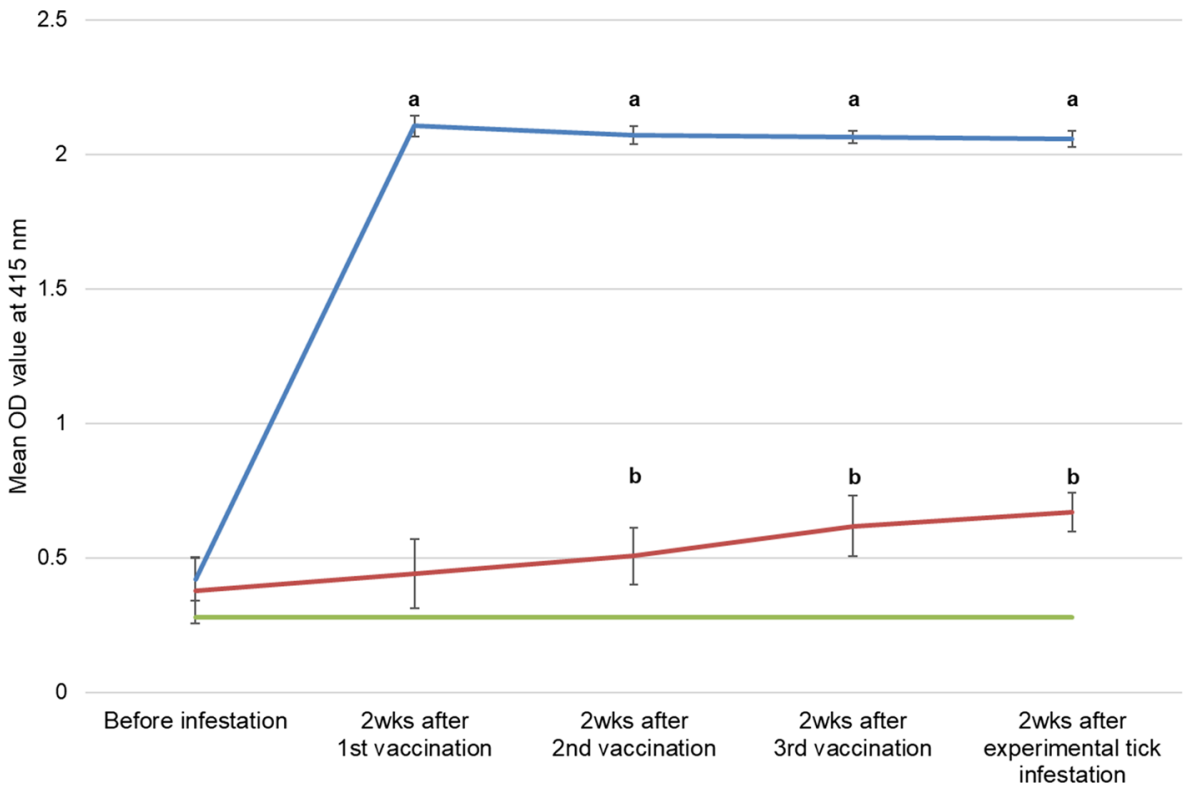

Fig. 3 Evaluation of antibody production after vaccination. The vaccination group and PBS group were established and tested. Antibody levels were estimated using ELISA, and the mean antibody titer is shown. Following each vaccination, there was a two-week interval before antibody levels were assessed. Statistical analysis regarding OD values between the two groups was performed using the Mann-Whitney test. a $P<0.005$ to $\mathrm{PBS}$ group; $\mathbf{b} P<0.005$ to naïve rabbit serum 

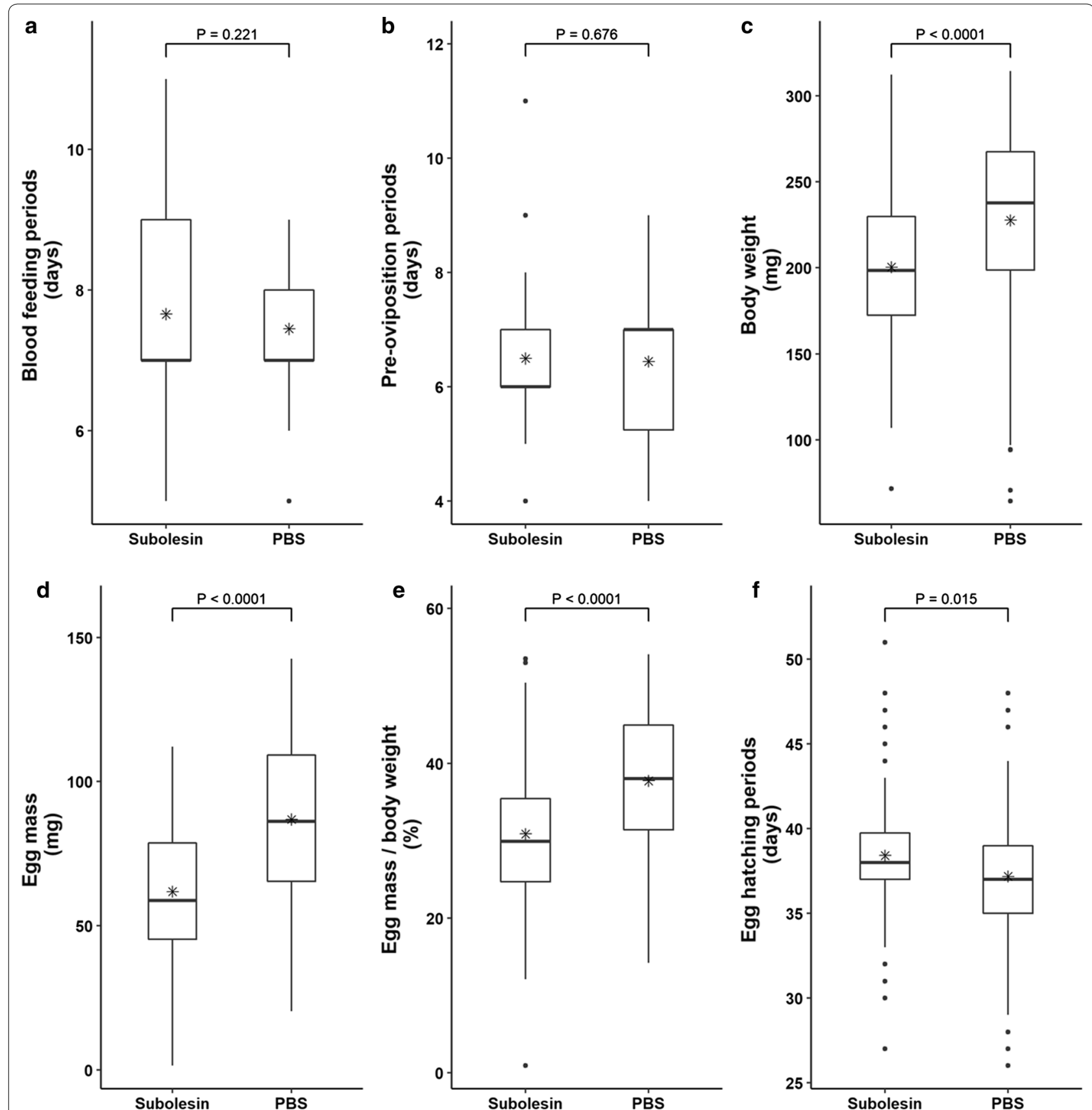

Fig. 4 Effect of rHISu vaccination on rabbits to control Haemaphysalis longicornis infestation. The results are shown as box plots. a Blood-feeding periods. $\mathbf{b}$ Pre-oviposition periods. c Body weight at engorgement. $\mathbf{d}$ Egg mass. e Egg mass to body weight ratio. $\mathbf{f}$ Egg-hatching periods. Mean values are indicated with an asterisk. Statistical comparison between the two groups for each parameter was performed using Student's t-test or Mann-Whitney test

knowledge, this is the first vaccination study of subolesin in Haemaphysalis spp., an important tick in Asian countries and an emerging tick in North America. Combined with the results of previous studies, this study suggests that subolesin has the potential to be used as a universal tick vaccine.

\section{Supplementary information}

Supplementary information accompanies this paper at https://doi. org/10.1186/s13071-020-04359-w.

Additional file 1: Table S1. Effect of rHISu vaccination on control of $H$. longicornis infestation in rabbits. Figure S1. Evaluation of antibody titers. 


\begin{abstract}
Abbreviations
ELISA: enzyme-linked immunosorbent assay; HISu: H. longicornis subolesin; ORF: open reading frame; PBS: phosphate-buffered saline; PBS-T: PBS containing $0.1 \%$ Tween 20; PCR: polymerase chain reaction; rHISu: recombinant HISu; SDS-PAGE: sodium dodecyl sulfate polyacrylamide gel electrophoresis; SFTS: severe fever with thrombocytopenia syndrome; TBS-T: TBS containing $0.1 \%$ Tween 20 .
\end{abstract}

\section{Acknowledgements}

The authors sincerely thank Dr Ragab M. Fereig, Ms Maki Kuniyori, and Mr Hiroyuki Sugawara for their technical support.

\section{Authors' contributions}

SHL, RUS and XX conceptualized the study. SHL, JL and RUS performed the rabbit experiments. SHL and JL performed ELISA and Western blotting. SHL drafted the initial manuscript. JL performed experiments for revision. SHL, RUS, $J$, PFAM, KO, WZ and ML performed the tick experiment. SHL, SJ and SK performed the statistical analyses. JL, SK, RUS and XX reviewed and contributed to the manuscript. XX supervised the study. All authors read and approved the final manuscript.

\section{Funding}

SHL was supported by a Research Fellowship for Young Scientists from the Japan Society for the Promotion of Science (JSPS) (ID No. P18089). This study was supported by a Grant-in-Aid for Scientific Research (18F18089, 18H02336) from Ministry of Education, Culture, Sports, Science and Technology (MEXT) of Japan, a grant JSPS Core-to-Core Programme, and a grant from MEXT of Japan as a project for Joint Usage/Research Center.

\section{Availability of data and materials}

The datasets supporting the conclusions of this article are included within the article and its additional files. The newly generated sequence was deposited in the GenBank database under the accession number MT199422.

\section{Ethics approval and consent to participate}

Prior to conducting the experiments, permission for the use of experimental animals was obtained from the Animal Care and Use Committee of Obihiro University of Agriculture and Veterinary Medicine, Obihiro, Hokkaido, Japan (approval no. 19-224 for rabbits and no. 19-74 for tick infestation using rabbits).

\section{Consent for publication}

Not applicable.

\section{Competing interests}

The authors declare that they have no competing interests.

\section{Author details}

${ }^{1}$ National Research Center for Protozoan Diseases, Obihiro University of Agriculture and Veterinary Medicine, Obihiro, Hokkaido 080-8555, Japan. ${ }^{2}$ College of Veterinary Medicine, Chungbuk National University, Cheongju 28644, South Korea.

Received: 14 April 2020 Accepted: 11 September 2020 Published online: 18 September 2020

\section{References}

1. Jongejan F, Uilenberg G. The global importance of ticks. Parasitology. 2004;129:S3-14.

2. Lee SH, Mossaad E, Ibrahim AM, Ismail AA, Moumouni PFA, Liu M, et al. Detection and molecular characterization of tick-borne pathogens infecting sheep and goats in Blue Nile and West Kordofan states in Sudan. Ticks Tick Borne Dis. 2018:9:598-604

3. Kim BJ, Kim H, Won S, Kim HC, Chong ST, Klein TA, et al. Ticks collected from wild and domestic animals and natural habitats in the Republic of Korea. Korean J Parasitol. 2014,52:281.

4. Shimada Y, Beppu T, Inokuma H, Okuda M, Onishi T. Ixodid tick species recovered from domestic dogs in Japan. Med Vet Entomol. 2003;17:38-45.

5. Raghavan RK, Barker SC, Cobos ME, Barker D, Teo EJM, Foley DH, et al. Potential spatial distribution of the newly introduced long-horned tick, Haemaphysalis longicornis in North America. Sci Rep. 2019;9:1-8.
6. Zhuang L, Sun Y, Cui XM, Tang F, Hu JG, Wang LY, et al. Transmission of severe fever with thrombocytopenia syndrome virus by Haemaphysalis longicornis ticks, China. Emerg Infect Dis. 2018;24:868.

7. Higuchi S, Simomura S, Yoshida H, Hoshi F, Kawamura S, Yasuda Y. Development of Babesia gibsoni in the hemolymph of the vector tick, Haemaphysalis longicornis. J Vet Med Sci. 1991;53:491-3.

8. Hatta T, Matsubayashi M, Miyoshi T, Islam MK, Alim MA, Yamaji K, et al. Quantitative PCR-based parasite burden estimation of Babesia gibsoni in the vector tick, Haemaphysalis longicornis (Acari: Ixodidae), fed on an experimentally infected dog. JVet Med Sci. 2013;75:1-6.

9. George JE, Pound JM, Davey RB. Chemical control of ticks on cattle and the resistance of these parasites to acaricides. Parasitology. 2004;129:5353-66.

10. Hayes EB. Is it time for a new yellow fever vaccine? Vaccine. 2010;28:8073-6.

11. Riley EM, Stewart VA. Immune mechanisms in malaria: new insights in vaccine development. Nat Med. 2013;19:168-78.

12. Nene V, Kiara H, Lacasta A, Pelle R, Svitek N, Steinaa L. The biology of Theileria parva and control of East Coast fever-current status and future trends. Ticks Tick Borne Dis. 2016;7:549-64.

13. Willadsen P, Riding GA, McKenna RV, Kemp DH, Tellam RL, Nielsen JN, et al. Immunologic control of a parasitic arthropod. Identification of a protective antigen from Boophilus microplus. J Immunol. 1989;143:1346-51.

14. de la Fuente J, Almazán C, Canales M, de la Lastra JMP, Kocan KM, Willadsen P. A ten-year review of commercial vaccine performance for control of tick infestations on cattle. Anim Health Res Rev. 2007;8:23-8.

15. Trentelman JJ, Teunissen $H$, Kleuskens JA, van de Crommert J, de la Fuente J, Hovius JW, et al. A combination of antibodies against Bm86 and Subolesin inhibits engorgement of Rhipicephalus australis (formerly Rhipicephalus microplus) larvae in vitro. Parasit Vectors. 2019;12:362.

16. Coumou J, Wagemakers A, Trentelman JJ, Nijhof AM, Hovius JW. Vaccination against Bm86 homologues in rabbits does not impair Ixodes ricinus feeding or oviposition. PLoS ONE. 2015:10:e0123495.

17. Rodríguez-Valle M, Taoufik A, Valdés M, Montero C, Hassan I, Hassan SM, et al Efficacy of Rhipicephalus (Boophilus) microplus Bm86 against Hyalomma dromedarii and Amblyomma cajennense tick infestations in camels and cattle. Vaccine. 2012;30:3453-8.

18. Liao M, Zhou J, Hatta T, Umemiya R, Miyoshi T, Tsuji N, et al. Molecular characterization of Rhipicephalus (Boophilus) microplus Bm86 homologue from Haemaphysalis longicornis ticks. Vet Parasitol. 2007;146:148-57.

19. Almazán C, Kocan KM, Bergman DK, Garcia-Garcia JC, Blouin EF, de la Fuente J. Identification of protective antigens for the control of Ixodes scapularis infestations using cDNA expression library immunization. Vaccine. 2003:21:1492-501

20. de la Fuente J, Almazán C, Blas-Machado U, Naranjo V, Mangold AJ, Blouin $\mathrm{EF}$, et al. The tick protective antigen, 4D8, is a conserved protein involved in modulation of tick blood ingestion and reproduction. Vaccine. 2006:24:4082-95.

21. Galindo RC, Doncel-Perez E, Zivkovic Z, Naranjo V, Gortazar C, Mangold AJ, et al. Tick subolesin is an ortholog of the akirins described in insects and vertebrates. Dev Comp Immunol. 2009:33:612-7.

22. Moreno-Cid JA, de la Lastra JMP, Villar M, Jiménez M, Pinal R, Estrada-Peña A, et al. Control of multiple arthropod vector infestations with subolesin/akirin vaccines. Vaccine. 2013;31:1187-96.

23. Merino O, Almazán C Canales M, Villar M, Moreno-Cid JA Galindo RC, et al. Targeting the tick protective antigen subolesin reduces vector infestations and pathogen infection by Anaplasma marginale and Babesia bigemina. Vaccine. 2011;29:8575-9.

24. Zivkovic Z, Torina A, Mitra R, Alongi A, Scimeca S, Kocan KM, et al. Subolesin expression in response to pathogen infection in ticks. BMC Immunol. 2010;11:7.

25. Bensaci M, Bhattacharya D, Clark R, Hu LT. Oral vaccination with vaccinia virus expressing the tick antigen subolesin inhibits tick feeding and transmission of Borrelia burgdorferi. Vaccine. 2012;30:6040-6.

26. Contreras M, de la Fuente J. Control of Ixodes ricinus and Dermacentor reticulatus tick infestations in rabbits vaccinated with the Q38 Subolesin/Akirin chimera. Vaccine. 2016;34:3010-3.

27. Rahman M, Saiful Islam M, You M. Impact of subolesin and cystatin knockdown by RNA interference in adult female Haemaphysalis longicornis (Acari: Ixodidae) on blood engorgement and reproduction. Insects. 2018;9:39.

28. Almazán C, Lagunes R, Villar M, Canales M, Rosario-Cruz R, Jongejan F, et al. Identification and characterization of Rhipicephalus (Boophilus) microplus 
candidate protective antigens for the control of cattle tick infestations Parasitol Res. 2010;106:471-9.

29. Nijhof AM, Taoufik A, De la Fuente J, Kocan KM, De Vries E, Jongejan F. Gene silencing of the tick protective antigens, Bm86, Bm91 and subolesin, in the one-host tick Boophilus microplus by RNA interference. Int J Parasitol. 2007;37:653-62.

30. Umemiya-Shirafuji R, Fujisaki K, Okado K, Moumouni PFA, Yokoyama N, Suzuki $H$, et al. Hard ticks as research resources for vector biology: from genome to whole-body level. Med Entomol Zool. 2019:70:181-8.

31. Umemiya-Shirafuji R, Matsuo T, Liao M, Boldbaatar D, Battur B, Suzuki HI, et al. Increased expression of ATG genes during nonfeeding periods in the tick Haemaphysalis longicornis. Autophagy. 2010;6:473-81.

32. Kamio T. The improvemetn of "ear bag" method for tick infestation. Proc Jpn Assoc Acarol. 1987;14:1-4 (In Japanese).
33. Prudencio CR, de la Lastra JMP, Canales M, Villar M, de la Fuente J. Mapping protective epitopes in the tick and mosquito subolesin ortholog proteins. Vaccine. 2010;28:5398-406.

34. Shakya M, Kumar B, Nagar G, de la Fuente J, Ghosh S. Subolesin: A candidate vaccine antigen for the control of cattle tick infestations in Indian situation. Vaccine. 2014;32:3488-94.

35. Merino O, Antunes S, Mosqueda J, Moreno-Cid JA, de la Lastra JMP Rosario-Cruz R, et al. Vaccination with proteins involved in tick-pathogen interactions reduces vector infestations and pathogen infection. Vaccine. 2013;31:5889-96.

\section{Publisher's Note}

Springer Nature remains neutral with regard to jurisdictional claims in published maps and institutional affiliations. 